\title{
ALMOST EVERYWHERE CONVERGENCE OF CONVOLUTION POWERS WITHOUT FINITE SECOND MOMENT
}

\author{
CHRISTOPHER M. WEDRYCHOWICZ
}

\begin{abstract}
Bellow and Calderón proved that the sequence of convolution powers $\mu_{n} f(x)=\sum_{k \in \mathbb{Z}} \mu^{n}(k) f\left(T^{k} x\right)$ converges a.e, when $\mu$ is a strictly aperiodic probability measure on $\mathbb{Z}$ such that the expectation is zero, $E(\mu)=0$, and the second moment is finite, $m_{2}(\mu)<\infty$. In this paper we extend this result to cases where $m_{2}(\mu)=\infty$.
\end{abstract}

\section{Almost everywhere convergence of Convolution powers}

1.1. Preliminaries. Let $\mu(k), k \in \mathbb{Z}$, be a probability measure. It's convolution $\mu * \mu$ is defined by $\mu * \mu(k)=\sum_{j \in \mathbb{Z}} \mu(j) \mu(k-j)$. The $n$-fold convolution $\mu * \cdots * \mu(k)=\mu^{n}(k)$ is defined inductively by $\mu^{n}(k)=\mu * \mu^{n-1}(k)$. The Fourier transform of $\mu$ will be denoted by $\theta(t)$ for $t \in[-1 / 2,1 / 2)$ and it is equal to

$$
\theta(t)=\sum_{k \in \mathbb{Z}} \mu(k) e^{2 \pi i k t}
$$

The weights may be recovered from the inversion formula

$$
\mu(k)=\int_{-1 / 2}^{1 / 2} \theta(t) e^{-2 \pi i k t} \mathrm{~d} t .
$$

The $p^{\text {th }}$ moment of $\mu$ is the sum $m_{p}(\mu)=\sum|k|^{p} \mu(k)$ and we say that $\mu$ has a $p^{\text {th }}$ moment if the above sum is finite. It will be necessary to consider positive non-integral moments $p$. The expectation of $\mu$ is given by $E(\mu)=\sum_{k \in \mathbb{Z}} k \mu(k)$.

Definition 1.1. $\mu$ is called strictly aperiodic if the support of $\mu$ is not contained in a proper coset of the integers.

We have the following important theorem by Foguel( [4).

Theorem 1.1. $\mu$ is strictly aperiodic $\Longleftrightarrow|\theta(t)| \neq 1 \forall t \neq 0$.

Now let $T: X \rightarrow X$ be an invertible measure preserving transformation of a probability space $(X, \mathcal{B}, \lambda)$. For $f \in \mathrm{L}^{1}(\lambda)$ one may define

$$
\mu f(x)=\sum_{k \in \mathbb{Z}} \mu(k) f\left(T^{k} x\right) .
$$

One then considers the question of a.e convergence for the sequence $\mu^{n} f(x)=\sum_{k \in \mathbb{Z}} \mu^{n}(k) f\left(T^{k} x\right)$. 
Definition 1.2. A probability measure $\mu$ on $\mathbb{Z}$ has bounded angular ratio, if $\mu$ is strictly aperiodic and

$$
1 \leq \sup _{t \neq 0} \frac{|\hat{\mu}(t)-1|}{1-|\hat{\mu}(t)|}<\infty
$$

Definition 1.3. We denote by $\mu^{n}: \mathrm{L}^{1}(X) \rightarrow \mathrm{L}^{1}(X)$ the operator defined by $\mu^{n} f(x)=\sum_{k \in \mathbb{Z}} \mu^{n}(k) f\left(T^{k} x\right)$. We refer to the sequence $\mu^{n}$ as the sequence of convolution powers of $\mu$.

The following Theorem establishes the necessity of of the bounded angular ratio condition in the study of convolution powers.

Theorem $1.4\left([\underline{6})\right.$. Suppose that $\mu$ is a probability measure on $\mathbb{Z}\left(\mu \neq \delta_{k}\right.$, i.e not concentrated in a single point) and that $\hat{\mu}$ has unbounded angular ratio. Then there exists a function $f \in L^{\infty}$ such that $\mu_{n} f(x)$ fails to converge a.e.

In order to establish some key properties of the measures under our consideration we will need the following Theorem from [9]; it provides a generalization of L'Hospital's rule concerning indeterminate forms of type $0 / 0$.

Theorem 1.5. Let $f(x)$ and $F(x)$ be continuous differentiable functions on an open interval $(a, x)$ where a may denote $-\infty$, and the differential coefficients $f^{\prime}$ and $F^{\prime}$ have no common zeros or infinities in the open interval and if, as $x$ approaches the value $a, f(x)$ and $F(x)$ each have the unique limit zero, then as $x$ approaches the value a the limits of

$$
\frac{f(x)}{F(x)}
$$

are the limits of

$$
\frac{f^{\prime}(x)}{F^{\prime}(x)}
$$

Lemma 1.6. Suppose $\mu$ is a probability measure defined on $\mathbb{Z}$ such that $m_{1}(\mu)<\infty$, $\mu$ has bounded angular ratio and $\theta^{\prime \prime}(t)$ exists on a set $S=(-\delta, \delta)-\{0\}$. Furthermore if $\theta(t)=f(t)+i g(t)$ we have $f^{\prime \prime}(t)<0$ on $S$ then

(a) $E(\mu)=0$,

(b) $\left|g^{\prime}(t)\right| \leq c_{1}\left|f^{\prime}(t)\right|$ and $\left|g^{\prime \prime}(t)\right| \leq c_{2}\left|f^{\prime \prime}(t)\right|$ on $S$.

Proof. (a) is a special case of a result proved in [3] (Proposition 1.9). To prove (b) we first note that by $[5$ (Lemma 1 )

$$
\limsup _{t \rightarrow 0} \frac{|1-\hat{\mu}(t)|}{1-|\hat{\mu}(t)|}<\infty \Leftrightarrow \limsup _{t \rightarrow 0}\left|\frac{g(t)}{1-f(t)}\right|<\infty
$$

Since $f^{\prime \prime}(t)<0$ on $S$ and $f^{\prime}(0)=0, f^{\prime}(t)$ has no zeros on $S$ except when $t=0$. Therefore by Theorem 1.5 the limit points of $\frac{g(t)}{1-f(t)}$ coincide with that of $\frac{g^{\prime}(t)}{-f^{\prime}(t)}$ and $\frac{g^{\prime \prime}(t)}{-f^{\prime \prime}(t)}$, which proves (b).

Definition 1.7. Given a sequence of operators $T_{n}: \mathrm{L}_{1} \rightarrow \mathcal{M}(X)$, where $\mathcal{M}(X)$ denotes the set of measurable functions, the maximal operator $T^{*}$ of $\left\{T_{n}\right\}$ is defined by $T^{*} f(x)=\sup _{n}\left|T_{n} f(x)\right|$. For the sequence $\left\{\mu^{n}\right\}$ the maximal operator will be denoted by $\mu^{*}$. 
Theorem 1.8. Let $(X, \mathcal{B}, m)$ be a probability space. If $\left\{T_{n}\right\}$ is a sequence of bounded operators such that

$$
T^{*} f(x)=\sup _{n}\left|T_{n} f(x)\right|<\infty \quad \text { a.e. }
$$

for every $f \in L_{1}$ then the set of functions in $L_{1}$ such that $T_{n} f(x)$ converges a.e. is closed.

In order to show $T^{*} f(x)<\infty$ a.e, one often establishes a weak maximal inequality for the operator $T^{*}$ of the form

$$
m\left(\left\{x: T^{*}(x) \geq \lambda\right\}\right) \leq C \frac{\|f\|_{1}}{\lambda}
$$

where $C$ is a constant independent of $f$ and $\lambda$.

Theorem 1.9 ( 3 ). If $\mu$ is strictly aperiodic, $\mu_{n} f(x)$ converges a.e. for all $f \in S=\left\{\left(f_{1} \circ T-f_{1}\right)+f_{2}: f_{1} \in L_{\infty}(X), f_{2}(T x)=f_{2}(x)\right.$ a.e. $\}$, which is a dense set in $L^{p}$ for all $p \geq 1$.

Remark 1.10. The above two Theorems imply that in order to establish a.e convergence of $\mu^{n} f(x)$ for a strictly aperiodic measure $\mu$ we need only show that $\mu^{*}$ satisfies a weak $\mathrm{L}_{1}$ inequality of the form given above.

In [1] it was shown that in order to establish weak maximal inequalities for operators on continuous spaces it is enough to establish them for an operator on $l^{1}(\mathbb{Z})$ that has been transfered from the continuous space. Such processes are known collectively as the Calderón Transfer Principle. In our case we make use of the following version.

Theorem 1.11 (Calderón Transfer Principle, 2]). Consider the dynamical system $(\mathbb{Z}, \mathbb{P},|\cdot|, T)$ where $|B|=$ \# of elements in $B, \mathbb{P}=$ Power set of $\mathbb{Z}$ and $T(x)=x+1$. For $\forall \phi \in l_{1}(\mathbb{Z})$ we have $\mu^{n} \phi(k)=\left(\mu^{n} * \phi\right)(k)=\sum_{j \in \mathbb{Z}} \mu^{n}(j) \phi(k-j)$ and $(M \phi)(k)=$ $\sup _{n}\left(\mu^{n} \phi\right)(k)$. Then if

$$
|k \in \mathbb{Z}:|(M \phi)(k)|\geq t| \leq C \frac{\|\phi\|_{l_{1}}}{t}
$$

we have

$$
\lambda(\{x \in X:|(M f)(x)| \geq t\}) \leq C \frac{\|f\|_{1}}{t}
$$

for all $(X, \mathcal{B}, \lambda, T)$ and for all $f \in L_{1}(X)$.

In light of the above, the following general result, which we shall use, was obtained in [1].

Theorem 1.12. Let $\left(\mu_{n}\right)$ be a sequence of probabilities on $\mathbb{Z}$ and for $\phi \in L_{1}(\mathbb{Z})$ define the maximal operator

$$
(M \phi)(x)=\sup _{n}\left|\left(\mu_{n} \phi\right)(x)\right|, \quad x \in \mathbb{Z}
$$

Assume that there is $0<\alpha \leq 1$ and $C^{\prime \prime}>0$ such that for each $n \geq 1$

$$
\left|\mu_{n}(x+y)-\mu_{n}(x)\right| \leq C^{\prime \prime} \frac{|y|^{\alpha}}{|x|^{1+\alpha}} \text {, for } x, y \in \mathbb{Z} \text {, and } 2|y| \leq|x|
$$


Then the maximal operator $M$ ie weak type $(1,1)$, i.e there is $C>0$ such that for any $\lambda>0$

$$
m\{x \in X:(M \phi)(x)>\lambda\} \leq \frac{C}{\lambda}\|f\|_{1} \text { for all } \phi \in L^{1}(X)
$$

Using the above, in [1, Bellow and Calderón established the a.e convergence of $\mu^{n} f(x)$ when $\mu$ is strictly aperiodic, $E(\mu)=0$ and $m_{2}(\mu)<\infty$. In [5] Losert shows that this result can not be extended to measures with $m_{p}(\mu)<\infty$ for $p<2$, however, in [3] it was shown that if $\mu$ is symmetric and $\mu(k) \geq \mu(k+1)$ for all $k>0$ then $\mu^{n} f(x)$ converges a.e $\forall f \in \mathrm{L}^{1}(X)$. This implies that the measure given by $\mu(k)=\frac{c}{|k| \log ^{2}|k|}$ yields a.e convergence of $\mu^{n} f(x)$ even though $m_{p}(\mu)=\infty$ for all $p>0$. The goal of this paper will be to extend the result in [1] by weakening the second moment condition.

Translating the second moment condition into a statement concerning Fourier transforms we obtain

$\mu$ has finite second moment $\Leftrightarrow \theta$ is twice continuously differentiable

Therefore we will seek a condition weaker than a continuous second derivative. The most obvious condition would be that $\theta^{\prime}(t) \in \operatorname{Lip}_{1}[-1 / 2,1 / 2]$, however the following ([7]) shows this extension to be vacuous,

Theorem 1.13 (Moricz). Let $f(x)=\sum_{k \in \mathbb{Z}} c_{k} e^{i k x}$. If $\left\{c_{k}\right\} \subset \mathbb{C}$ is such that $\sum_{|k| \leq n}\left|k c_{k}\right|=$ $O\left(n^{1-\alpha}\right), n=1,2, \ldots$, for some $0<\alpha \leq 1$, then $f \in \operatorname{Lip}(\alpha)$. Conversely, let $c_{k}$ be a sequence of real numbers such that $k c_{k} \geq 0$ for all $k \in \mathbb{Z}$. If $\sum_{k \in \mathbb{Z}}\left|c_{k}\right|$ is finite and $f \in \operatorname{Lip}(\alpha)$ for some $0<\alpha \leq 1$, then $\sum_{|k| \leq n}\left|k c_{k}\right|=O\left(n^{1-\alpha}\right)$.

Although the extension $\theta^{\prime}(t) \in \operatorname{Lip}(1)$ is vacuous, the condition $\theta^{\prime}(t) \in \operatorname{Lip}(\alpha)$ for some $0<\alpha<1$ will not be. We will construct examples of non-symmetric measures $\mu$ with $m_{2}(\mu)=\infty$ with $\left\{\mu^{n} f(x)\right\}$ converging a.e. In fact, given $p>1$, we will give examples of non-symmetric $\mu$ with $E(\mu)=0, m_{p}(\mu)=\infty$ and $\left\{\mu^{n} f(x)\right\}$ converging a.e.

We will need the following.

Theorem 1.14. (8]) Let $\theta(t)$ be the Fourier transform of a measure $\mu$ on $\mathbb{Z}$ not supported at a single integer. Then there exist positive constants $\delta$ and $\epsilon$ such that $|\theta(t)| \leq 1-\epsilon t^{2}$ for $|t| \leq \delta$. Therefore for $\mu$ strictl $y$ aperiodic there exists a $C$ such that $|\theta(t)| \leq e^{-C t^{2}} \forall t \in[-1 / 2,1 / 2)$.

Lemma 1.15. (1]) There is a constant $C>0$ such that, for any $x, y \in \mathbb{Z}, 0<$ $2|y|<|x|$, and $t \in \mathbb{R}$

$$
\left|\frac{e((x+y) t)-1}{(x+y)^{2}}-\frac{e(x t)-1}{x^{2}}\right| \leq C|t| \frac{|y|}{|x|^{2}},
$$

where $e(x)=e^{2 \pi i x}$.

This paper contains results from the author's Ph.D dissertation. He would like to thank his adviser, Dr. Karin Reinhold, for all her help and insightful comments throughout the process. The author would also like to thank the referee for the 
insightful comments and suggestions. In particular that of the use of the bounded angular ratio condition which appears throughout this paper, and how it could be used in the proof of Proposition 2.7.

\section{Main Results}

Throughout we suppose that all measures $\mu$ have bounded angular ratio and $m_{1}(\mu)<\infty$. Note that a constant $c$, independent of certain quantities, may change throughout an argument. Our main results are the following.

Theorem 2.1. Suppose $\mu$ is a probability on $\mathbb{Z}$ with $m_{1}(\mu)<\infty$ and bounded angular ratio and for some $0<\alpha \leq 1 \sum_{|k| \leq n} k^{2} \mu(k)=O\left(n^{1-\alpha}\right)$. Suppose $\theta^{\prime \prime}(t)$ exists in some set $0<|t|<\delta$ and $\operatorname{Re}\left(\theta^{\prime \prime}(t)\right)=p(t)+O(1)<0$, where $p(t)$ is non-decreasing in this set. Then $\left\{\mu^{n} f(x)\right\}$ converges a.e. for all $f \in L^{1}(X)$.

Since symmetric, strictly aperiodic measures have real-valued Fourier transform they have bounded angular ratio 1 . Hence, we have the following corollary.

Corollary 2.2. Suppose $\mu$ is a strictly aperiodic, symmetric measure on $\mathbb{Z}$ and for some $0<\alpha \leq 1 \sum_{|k| \leq n} k^{2} \mu(k)=O\left(n^{1-\alpha}\right)$. Suppose $\theta^{\prime \prime}(t)$ exists in some set $0<|t|<\delta$, and $\theta^{\prime \prime}(t)=p(t)+O(1)<0$, where $p(t)$ is non decreasing in this set. Then $\left\{\mu^{n} f(x)\right\}$ converges a.e. for all $f \in L^{1}(X)$.

The following gives examples of non symmetric measures $\mu$ with $m_{2}(\mu)=\infty$ and $\mu_{n} f(x)$ converging a.e. for all $f \in \mathrm{L}^{1}(X)$.

Example 2.3. Let $\eta(k)=s /|k|^{3}, k \neq 0$ where $s=\left(\sum 1 /\left(|k|^{3}\right)\right)^{-1}$. Then $\hat{\eta}(t)=\sum_{k>0} \frac{s}{|k|^{3}} \cos (2 \pi k t)$ and therefore $\hat{\eta}^{\prime \prime}(t)=-4 \pi^{2} s \sum_{k>0} 1 / k \cos (2 \pi k t)$. We have $\sum_{|k| \leq n} 1 /|k|=O(\log (n))$ and $\sum \frac{1}{k} \cos (2 \pi k t)=\log \left(\frac{1}{|2 \sin (x / 2)|}\right)$, (see [10]). Hence, $\eta^{\prime \prime}(t)=-4 \pi^{2} s \log \left(\frac{1}{|2 \sin (t / 2)|}\right)$ is monotone in a neighborhood of 0 . If $\nu$ is a measure with $E(\nu)=0$ and $m_{2}(\nu)<\infty$ then $\mu=a_{1} \eta+\left(1-a_{1}\right) \nu$ with $0<a_{1} \leq 1$, will have $\theta^{\prime \prime}(t)=p(t)+O(1)(t)$ and by Theorem $2.1\left(\mu^{n} f\right)(x)$ converges a.e. Note here that $m_{p}(\mu)$ is finite if and only if $p<2$.

Example 2.4. Let $\eta(k)=s /|k|^{2+\sigma}$ for some $0<\sigma<1, k \neq 0$ and $s=$ $\left(\sum 1 /|k|^{2+\sigma}\right)^{-1}$. Since $\sum_{|k| \leq n} 1 /|k|^{\sigma}=O\left(n^{1-\sigma}\right)$ and $\hat{\eta}^{\prime \prime}(t)=$

$\sum_{k=1}^{\infty} \frac{\cos (2 \pi k t)}{k^{\alpha}}=\Gamma(1-\alpha) \sin \left(\frac{1}{2} \pi \alpha\right) t^{\alpha-1}+O(1)$, (see [10]). Then we can construct a measure $\mu=a_{1} \eta+\left(1-a_{1}\right) \nu$ where $0<a_{1} \leq 1$ and $\nu$ is as in the previous example such that $\mu_{n} f(x)$ converges a.e. Note here that $m_{p}(\mu)$ is finite if and only if $p<1+\sigma$. 
Proposition 2.5. Suppose that the measure $\mu$ satisfies the condition $\sum_{|k| \leq n} k^{2} \mu(k)=$ $O\left(n^{1-\delta}\right)$, so that the Fourier transform $\theta(t)$ has $\theta^{\prime}(t) \in \operatorname{Lip} p_{\delta}$, for some $0<\delta \leq 1$, then

$$
\left|\mu^{n}(x)\right| \leq c\left\{\frac{\sqrt{n}}{|x|^{1+\delta}}+\frac{n^{2}}{|x|^{2}}\right\}
$$

Proof.

$$
\begin{aligned}
\left|\mu^{n}(x)\right| & =\left|\int_{-1 / 2}^{1 / 2} \theta^{n}(t) e^{-2 \pi i x t} \mathrm{~d} t\right|, \quad \text { by integration by parts } \\
& =\frac{1}{2 \pi}\left|\frac{n}{x} \int_{-1 / 2}^{1 / 2} \theta^{n-1}(t) \theta^{\prime}(t) e^{-2 \pi i x t} \mathrm{~d} t\right| \\
& \leq c \frac{n}{|x|}\left|\int_{-1 / 2}^{1 / 2}\left(\theta^{n-1}(t+h) \theta^{\prime}(t+h)-\theta^{n-1}(t) \theta^{\prime}(t)\right) e^{-2 \pi i x t} \mathrm{~d} t\right|, \quad \text { where } h=\frac{1}{2 x} \\
& \leq c \frac{n}{|x|} \int_{-1 / 2}^{1 / 2}\left|\theta^{n-1}(t+h)\right|\left|\theta^{\prime}(t+h)-\theta^{\prime}(t)\right|+\left|\theta^{\prime}(t)\right|\left|\theta^{n-1}(t+h)-\theta^{n-1}(t)\right| \mathrm{d} t \\
& =c \frac{n}{|x|} I_{1}+c \frac{n}{|x|} I_{2} .
\end{aligned}
$$

Now we examine $I_{1}$ and $I_{2}$ separately. By the Lipschitz property of $\theta^{\prime}(t)$

$$
\begin{aligned}
I_{1} & =\frac{1}{x^{\delta}} \int_{-1 / 2}^{1 / 2}\left|\theta^{n-1}(t+h)\right| x^{\delta}\left|\theta^{\prime}(t+h)-\theta^{\prime}(t)\right| \mathrm{d} t \\
& \leq \frac{c}{x^{\delta}} \int_{-1 / 2}^{1 / 2}\left|\theta^{n-1}(t+h)\right| \mathrm{d} t \\
& \leq \frac{c}{x^{\delta}} \frac{1}{\sqrt{n}}, \quad \text { by } 1.14 .
\end{aligned}
$$

Therefore,

$$
\begin{gathered}
\frac{c n}{|x|} I_{1} \leq \frac{c \sqrt{n}}{x^{1+\delta}}, \\
I_{2}=\frac{1}{|x|} \int_{-1 / 2}^{1 / 2}\left|\theta^{\prime}(t)\right||x|\left|\theta^{n-1}(t+h)-\theta^{n-1}(t)\right| \mathrm{d} t \\
\leq c \frac{n-1}{|x|} \int_{-1 / 2}^{1 / 2}\left|\theta^{\prime}(t)\right|\left|\theta^{\prime}(c(t))\right|\left|\theta^{n-2}(c(t))\right| \mathrm{d} t \leq \frac{c n}{|x|}
\end{gathered}
$$

for some $c(t)$ between $t$ and $t+h$ by the mean value theorem.

Therefore

$$
\frac{c n}{|x|} I_{2} \leq \frac{c n^{2}}{|x|^{2}}
$$

Hence

$$
\left|\mu_{n}(x)\right| \leq \frac{c_{1} \sqrt{n}}{|x|^{1+\delta}}+\frac{c_{2} n^{2}}{|x|^{2}} .
$$


Corollary 2.6. Let $\sigma=\min \left\{\frac{15 \delta}{16}, \frac{3}{4}\right\}$ then $\sigma>0$ and if $n \leq|x|^{\frac{\delta}{8}}$,

$$
\left|\mu_{n}(x)\right| \leq \frac{C}{|x|^{1+\sigma}} \leq \frac{C|y|^{\sigma}}{|x|^{1+\sigma}}
$$

$\forall y \in \mathbb{Z}, y \neq 0$.

Proposition 2.7. Suppose there is a set $(-\delta, \delta)-\{0\}$ on which $\theta(t)$ is twice differentiable and assume that if $\theta(t)=f(t)+i g(t)$, we have $f^{\prime \prime}(t)=p(t)+O(1)<0$ where $p(t)$ is non-decreasing on $(-\delta, \delta)-\{0\}$ and $p(t) \rightarrow-\infty$ as $t \rightarrow 0$. Then, for $t$ in $(-\delta, \delta)$, there exists a positive constant $c$ such that

$$
|\theta(t)| \leq 1-c \phi(t) t^{2}
$$

where $\phi(t)=\left|\frac{f^{\prime}(t)}{t}\right|$.

Proof. By bounded angular ratio

$$
\begin{aligned}
|\theta(t)| & \leq 1-c|1-\theta(t)| \\
& \leq 1-c|1-f(t)| .
\end{aligned}
$$

Since $f^{\prime \prime}(t)<0, f^{\prime}(0)=0$ and $p(t) \rightarrow-\infty$ implies $\left|\frac{f^{\prime}(t / 2)}{f^{\prime}(t)}\right|$ is bounded below, we have that the above quantity

$$
\begin{aligned}
& =1-c\left|\int_{0}^{t} f^{\prime}(s) \mathrm{d} s\right| \\
& \leq 1-c\left|\int_{t / 2}^{t} f^{\prime}(s) \mathrm{d} s\right| \\
& \leq 1-c \frac{|t|}{2}\left|f^{\prime}(t / 2)\right| \\
& \leq 1-c^{\prime} t^{2}\left|\frac{f^{\prime}(t)}{t}\right| .
\end{aligned}
$$

Lemma 2.8. Suppose that $\operatorname{Re}\left(\theta^{\prime \prime}(t)\right)=f^{\prime \prime}(t)=p(t)+O(1)<0$ on a set $S=$ $(-\delta, \delta)-\{0\}$ where $p(t)$ is non-increasing as $|t| \rightarrow 0$, and $p(t) \rightarrow-\infty$ as $t \rightarrow 0$. Then the function defined by $\phi(t)=\left|\frac{f^{\prime}(t)}{t}\right|$ satisfies the following properties for all $t$ in some set $\left[-\delta^{\prime}, \delta^{\prime}\right]-\{0\}$,

(1) $\phi(t)=\phi(-t)$

(2) There exist constants $2>c_{1}>1, c_{2}>0, c_{3}>0$ such that $c_{1} \phi(t) \geq\left|f^{\prime \prime}(t)\right|$, $c_{2} \phi(t) \geq\left|\theta^{\prime \prime}(t)\right|, c_{3} \phi(t) \geq\left|\frac{\theta^{\prime}(t)}{t}\right|$

(3) $|t \phi(t)| \rightarrow 0$ as $t \rightarrow 0$ and $\left|t \phi^{\prime}(t)\right| \leq \phi(t)$

Proof. We have $f(t)=\sum_{k \geq 0} c_{k} \cos (2 \pi k t)$ with $c_{k} \geq 0$ and therefore the first assertion is trivial. Since $f^{\prime}(0)=0,|t \phi(t)| \rightarrow 0$. We have, as $f^{\prime}(0)=0$ for $c(t)$ between 0 
and $t$,

$$
\begin{aligned}
\phi(t) & =\left|\frac{f^{\prime}(t)}{t}\right|=\left|f^{\prime \prime}(c(t))\right|=|p(c(t))+O(1)| \\
& \geq|p(t)+O(1)|
\end{aligned}
$$

Hence for some $2>c_{1}>1$, since $\phi(t) \rightarrow \infty$ as $t \rightarrow 0$,

$$
\begin{aligned}
c_{1} \phi(t) & \geq|p(t)|+\left(c_{1}-1\right) \phi(t)-|O(1)| \\
& \geq|p(t)|+|O(1)| \geq|p(t)+O(1)| \\
& =\left|f^{\prime \prime}(t)\right| .
\end{aligned}
$$

Since by 1.6 (b) $\left|g^{\prime \prime}(t)\right| \leq c\left|f^{\prime \prime}(t)\right|$, we have $\left|g^{\prime \prime}(t)\right| \leq c^{\prime} \phi(t)$. So $\left|\theta^{\prime \prime}(t)\right| \leq\left|g^{\prime \prime}(t)\right|+$ $\left|f^{\prime \prime}(t)\right| \leq c_{2} \phi(t)$. Observe that, by 1.6 (b)

$$
\begin{aligned}
\left|\frac{\theta^{\prime}(t)}{t}\right| & \leq\left|\frac{g^{\prime}(t)}{t}\right|+\left|\frac{f^{\prime}(t)}{t}\right| \\
& \leq c\left|\frac{f^{\prime}(t)}{t}\right|+\left|\frac{f^{\prime}(t)}{t}\right| \\
& =c \phi(t) .
\end{aligned}
$$

Therefore the second assertion has been established. Now $\phi(t)=-\frac{f^{\prime}(t)}{t}$ so $\phi^{\prime}(t)=$ $\frac{-f^{\prime \prime}(t) t+f^{\prime}(t)}{t^{2}}$ and $t \phi^{\prime}(t)=-f^{\prime \prime}(t)+\frac{f^{\prime}(t)}{t}$ which implies

$$
\begin{aligned}
\left|t \phi^{\prime}(t)\right| & =\left|\frac{f^{\prime}(t)}{t}-f^{\prime \prime}(t)\right| \\
& =|| f^{\prime \prime}(t)|-| \frac{f^{\prime}(t)}{t}|| \\
& \leq|2 \phi(t)-\phi(t)|=\phi(t) .
\end{aligned}
$$

Thus the third assertion follows.

Lemma 2.9. For a function $\phi(t)$ satisfying the properties of Lemma 2.8, the following hold.

(1)

$$
n \int_{-\delta}^{\delta}\left(1-k t^{2} \phi(t)\right)^{n-1}|t| \phi(t)<C
$$

$$
n^{2} \int_{-\delta}^{\delta}\left(1-k t^{2} \phi(t)\right)^{n-2}|t|^{3} \phi^{2}(t)<C
$$

where $C$ is independent of $n$, and $0 \leq 1-k t^{2} \phi(t) \leq 1$ on $(-\delta, \delta)$. 
ALMOST EVERYWHERE CONVERGENCE OF CONVOLUTION POWERS WITHOUT FINITE SECOND MOMENT

Proof.

$$
\begin{aligned}
n \int_{-\delta}^{\delta}\left(1-k t^{2} \phi(t)\right)^{n-1}|t| \phi(t) \mathrm{d} t & =2 n \int_{0}^{\delta}\left(1-k t^{2} \phi(t)\right)^{n-1} t \phi(t) \mathrm{d} t \\
& =\frac{2 n}{-2 k} \int_{0}^{\delta}\left(1-k t^{2} \phi(t)\right)^{n-1}(-2 k t \phi(t)) \mathrm{d} t \\
& =\frac{n}{-k} \int_{0}^{\delta}\left(1-k t^{2} \phi(t)\right)^{n-1}\left(-2 k t \phi(t)-k t^{2} \phi^{\prime}(t)\right) \mathrm{d} t \\
& +\left(\frac{n}{k}\right) \int_{0}^{\delta}\left(1-k t^{2} \phi(t)\right)^{n-1}\left(-k t^{2} \phi^{\prime}(t)\right) \mathrm{d} t .
\end{aligned}
$$

Therefore

$$
\begin{aligned}
2 n \int_{0}^{\delta}\left(1-k t^{2} \phi(t)\right)^{n-1} t \phi(t) \mathrm{d} t & -\frac{n}{k} \int_{0}^{\delta}\left(1-k t^{2} \phi(t)\right)^{n-1}\left(-k t^{2} \phi^{\prime}(t)\right) \mathrm{d} t= \\
& =-\frac{n}{k} \int_{0}^{\delta}\left(1-k t^{2} \phi(t)\right)^{n-1}\left(-2 k t \phi(t)-k t^{2} \phi^{\prime}(t)\right) \mathrm{d} t \\
& =\frac{n}{k} \int_{1-k \delta^{2} \phi(\delta)}^{1} u^{n-1} \mathrm{~d} u \leq C .
\end{aligned}
$$

However, since $\left|t \phi^{\prime}(t)\right| \leq \phi(t)$ we have

$$
\begin{aligned}
2 n \int_{0}^{\delta}\left(1-k t^{2} \phi(t)\right)^{n-1} t \phi(t) \mathrm{d} t & +n \int_{0}^{\delta}\left(1-k t^{2} \phi(t)\right)^{n-1} t^{2} \phi^{\prime}(t) \mathrm{d} t \\
& \geq n \int_{0}^{\delta}\left(1-k t^{2} \phi(t)\right)^{n-1} t \phi(t) \mathrm{d} t \\
& =\frac{1}{2} n \int_{-\delta}^{\delta}\left(1-k t^{2} \phi(t)\right)^{n-1}|t| \phi(t) \mathrm{d} t .
\end{aligned}
$$

The claim follows.

$(2)$

$$
\begin{aligned}
& n^{2} \int_{-\delta}^{\delta}\left(1-k t^{2} \phi(t)\right)^{n-2}|t|^{3} \phi^{2}(t) \mathrm{d} t= \\
& =2 n^{2} \int_{0}^{\delta}\left(1-k t^{2} \phi(t)\right)^{n-2} t^{3} \phi^{2}(t) \mathrm{d} t \\
& =2 n^{2} \int_{0}^{\delta}\left(1-k t^{2} \phi(t)\right)^{n-2} t \phi(t) t^{2} \phi(t) \mathrm{d} t \\
& =\frac{n^{2}}{-k^{2}} \int_{0}^{\delta}\left(1-k t^{2} \phi(t)\right)^{n-2}\left(-2 k t \phi(t)-k t^{2} \phi^{\prime}(t)\right) t^{2} \phi(t) \mathrm{d} t \\
& \quad+\left(\frac{n^{2}}{k}\right) \int_{0}^{\delta}\left(1-k t^{2} \phi(t)\right)^{n-2}\left(-k t^{2} \phi^{\prime}(t)\right) t^{2} \phi(t) \mathrm{d} t
\end{aligned}
$$


Therefore by similar arguments as in the first part of this lemma, we have,

$$
\begin{aligned}
\mid \frac{1}{2} & n^{2} \int_{-\delta}^{\delta}\left(1-k t^{2} \phi(t)\right)^{n-2} t^{3} \phi^{2}(t) \mathrm{d} t \mid \leq \\
\leq & -\frac{n^{2}}{k} \int_{0}^{\delta}\left(1-k t^{2} \phi(t)\right)^{n-2}\left(-2 k t \phi(t)-k t^{2} \phi^{\prime}(t)\right) t^{2} \phi(t) \mathrm{d} t \\
& =\frac{n^{2}}{k} \int_{1-k \delta^{2} \phi(\delta)}^{1} u^{n-2}(1-u) \mathrm{d} u \\
& =\frac{n^{2}}{k^{2}} \int_{1-k \delta^{2} \phi(\delta)}^{1} u^{n-2}-u^{n-1} \mathrm{~d} u \\
& =\frac{n^{2}}{k^{2}}\left(\frac{u^{n-1}}{n-1}-\left.\frac{u^{n}}{n}\right|_{1-k \delta^{2} \phi(\delta)} ^{1}\right) \\
& =\frac{n^{2}}{k^{2}}\left(\frac{1}{n-1}-\frac{1}{n}+\frac{\left(1-k \delta^{2} \phi(\delta)\right)^{n}}{n}-\frac{\left(1-k \delta^{2} \phi(\delta)\right)^{n-1}}{n-1}\right) \\
\leq & \frac{n^{2}}{k^{2}}\left(\frac{1}{(n-1) n}+O\left(\frac{\left(1-k \delta^{2} \phi(\delta)\right)^{n}}{n}\right)\right) \\
\leq C & \frac{n^{2}}{k^{2}}\left(\frac{1}{n^{2}}+\frac{1}{n} O\left(\left(1-k \delta^{2}\right)^{n}\right)\right) \\
\leq & C \frac{n^{2}}{k^{2}}\left(\frac{1}{n^{2}}+\frac{1}{n} O\left(e^{-n k \delta^{2}}\right)\right) \\
\leq C & \frac{n^{2}}{k^{2}}\left(\frac{1}{n^{2}}+O\left(\frac{1}{n^{3}}\right)\right) \leq C
\end{aligned}
$$

Theorem 2.10. Suppose that $\theta(t)$ is twice differentiable in a neighborhood $[-\delta, \delta]$ of 0 , except perhaps at 0 , and there exists a function $\phi(t)$ satisfying the properties (1) - (3) of Lemma 2.8 such that

(1) $|\theta(t)| \leq 1-k t^{2} \phi(t)$

(2) $\left|\frac{\theta^{\prime}(t)}{t}\right| \leq c \phi(t)$

(3) $\left|\theta^{\prime \prime}(t)\right| \leq c \phi(t)$

on $[-\delta, \delta]$ for some $k, c>0$, then

$$
\left|\mu_{n}(x+y)-\mu_{n}(x)\right| \leq c \frac{|y|}{|x|^{2}} \quad \text { for } n \geq|x|^{\delta / 8} \text { and }|y| \leq \frac{|x|}{2}
$$

Remark 2.11. If $\theta^{\prime \prime}(t)$ is bounded on some $[-\delta, \delta]$ then the function $\phi(t)=$ $\sup _{t \in[-\delta, \delta]}\left\{\left|\theta^{\prime \prime}(t)\right|\right\}$ satisfies preporties (1) - (3) of Lemma2.8 as well as (1) - (3) of Theorem 2.10. Thus Theorem 2.10 applies to this case. Note that (1) of Theorem 2.10 follows from Theorem 1.14 
Proof of Theorem 2.10. By the inversion formula

$$
\begin{aligned}
\mu_{n}(x) & =\int_{-1 / 2}^{1 / 2} \theta^{n}(t) e^{-2 \pi i x t} \mathrm{~d} t \\
& =\int_{|t| \leq \delta} \theta^{n}(t) e^{-2 \pi i x t} \mathrm{~d} t+\int_{|t| \geq \delta} \theta^{n}(t) e^{-2 \pi i x t} \mathrm{~d} t \\
& =I_{1, x}+I_{2, x}
\end{aligned}
$$

Now, if $\theta^{n}(t)=\theta_{n}(t)$ we note that since $\theta^{\prime}(t)=\int_{\epsilon}^{t} \theta^{\prime \prime}(t)+\theta^{\prime}(\epsilon)$, letting $\epsilon \rightarrow 0$ we have $\theta^{\prime}(t)=\int_{0}^{t} \theta^{\prime \prime}(t)$ and $\theta^{\prime}(t)$ is absolutely continuous on $[-\delta, \delta]$ hence

$$
\begin{aligned}
I_{1, x} & =\frac{1}{2 \pi i}\left(-\left.\frac{\theta_{n}(t) e^{-2 \pi i x t}}{x}\right|_{-\delta} ^{\delta}+\frac{1}{x} \int_{-\delta}^{\delta} \theta_{n}^{\prime}(t) e^{-2 \pi i x t} \mathrm{~d} t\right) \\
& =\frac{1}{2 \pi i}\left(-\left.\frac{\theta_{n}(t) e^{-2 \pi i x t}}{x}\right|_{-\delta} ^{\delta}\right)+\frac{1}{4 \pi^{2}}\left(\left.\frac{\theta_{n}^{\prime}(t) e^{-2 \pi i x t}}{x^{2}}\right|_{-\delta} ^{\delta}\right) \\
& -\frac{1}{4 \pi^{2}}\left(\frac{1}{x^{2}} \int_{-\delta}^{\delta} \theta_{n}^{\prime \prime}(t) e^{-2 \pi i x t} \mathrm{~d} t\right) \\
& =Q_{x}-\frac{1}{4 \pi^{2} x^{2}} \int_{-\delta}^{\delta} \theta_{n}^{\prime \prime}(t) e^{-2 \pi i x t} \mathrm{~d} t \\
=Q_{x} & -\frac{1}{4 \pi x^{2}} \int_{-\delta}^{\delta} \theta_{n}^{\prime \prime}(t)\left(e^{-2 \pi i x t}-1\right) \mathrm{d} t-\frac{1}{4 \pi x^{2}} \int_{-\delta}^{\delta} \theta_{n}^{\prime \prime}(t) \mathrm{d} t \\
=Q_{x} & -\frac{1}{4 \pi^{2} x^{2}}\left(\theta_{n}^{\prime}(\delta)-\theta_{n}^{\prime}(-\delta)\right)-\frac{1}{4 \pi^{2} x^{2}} \int_{-\delta}^{\delta} \theta_{n}^{\prime \prime}(t)\left(e^{-2 \pi i x t}-1\right) \mathrm{d} t \\
=Q_{x} & +P_{x}-\frac{1}{4 \pi^{2} x^{2}} \int_{-\delta}^{\delta} \theta_{n}^{\prime \prime}(t)\left(e^{-2 \pi i x t}-1\right) \mathrm{d} t .
\end{aligned}
$$

Therefore

$$
\mu_{n}(x)=-\frac{1}{4 \pi^{2} x^{2}} \int_{-\delta}^{\delta} \theta_{n}^{\prime \prime}(t)\left(e^{-2 \pi i x t}-1\right) \mathrm{d} t+Q_{x}+P_{x}+I_{2, x}
$$

We have, for $2|y| \leq|x|$ by Lemma 1.15 . 


$$
\begin{aligned}
& \left|\int_{-\delta}^{\delta} \theta_{n}^{\prime \prime}(t) \frac{e^{-2 \pi i(x+y) t}-1}{(x+y)^{2}} \mathrm{~d} t-\int_{-\delta}^{\delta} \theta_{n}^{\prime \prime}(t) \frac{e^{-2 \pi i x t}-1}{x^{2}} \mathrm{~d} t\right| \\
& \quad \leq C \frac{|y|}{|x|^{2}} \int_{-\delta}^{\delta}\left|\theta_{n}^{\prime \prime}(t)\right||t| \mathrm{d} t \text { recalling that } \theta_{n}^{\prime \prime}(t)=\left(\theta^{n}(t)\right)^{\prime \prime} \text { and by 1.14 and } 2.7 \\
& \quad \leq C \frac{|y|}{|x|^{2}}\left[n(n-1) \int_{-\delta}^{\delta}\left|\theta^{n-2}(t)\right|\left|\theta^{\prime}(t)\right|^{2}|t| \mathrm{d} t+n \int_{-\delta}^{\delta}\left|\theta^{n-1}(t)\right|\left|\theta^{\prime \prime}(t)\right||t| \mathrm{d} t\right] \\
& \quad \leq C \frac{|y|}{|x|^{2}}\left(n^{2} \int_{-\delta}^{\delta}\left(1-k t^{2} \phi(t)\right)^{n-2}|t|^{3} \phi^{2}(t) \mathrm{d} t+n \int_{-\delta}^{\delta}\left(1-k t^{2} \phi(t)\right)^{n-1} \phi(t)|t| \mathrm{d} t\right) \\
& \quad \leq C \frac{|y|}{|x|^{2}} .
\end{aligned}
$$

Since $\left|\theta^{n}(t)\right| \leq e^{-C n t^{2}},\left|\theta^{n}(-\delta)\right|,\left|\theta^{n}(\delta)\right| \leq e^{-C n \delta^{2}}$ and $\left|\theta^{\prime}(\delta)\right|,\left|\theta^{\prime}(-\delta)\right|=\left|n \theta^{n-1}(\delta) \theta^{\prime}(\delta)\right|,\left|n \theta^{n-1}(-\delta) \theta^{\prime}(-\delta)\right| \leq C n e^{-n \delta^{2}}$, we have $\left|I_{2, x}\right|,\left|P_{x}\right|,\left|Q_{x}\right| \leq$ $C n e^{-n \delta^{2}} \leq \frac{C}{n^{16 / \delta}} \leq \frac{c}{|x|^{2}}$ provided that $n \geq|x|^{\delta / 8}$. Since $y \in \mathbb{Z}$ and $|y| \leq \frac{|x|}{2}$ we have

$$
\left|I_{2, x+y}-I_{2, x}\right|,\left|P_{x+y}-P_{x}\right|,\left|Q_{x+y}-Q_{x}\right| \leq \frac{c}{|x|^{2}} \leq C \frac{|y|}{|x|^{2}} .
$$

The theorem follows.

Combining 2.6, 2.7 2.8 and 2.10 we obtain.

Theorem 2.12. Let $\mu$ be a probability with $m_{1}(\mu)<\infty$, bounded angular ratio and $\sum_{|k|<n} k^{2} \mu(k)=O\left(n^{1-\delta}\right)$ for some $0<\delta \leq 1$. Suppose $\theta(t)=f(t)+i g(t)$ is the Fourier transform of $\mu$ and $\theta^{\prime \prime}(t)$ exists at all points except possibly 0 in a neighborhood $[-\delta, \delta]$. Then if $f^{\prime \prime}(t)=p(t)+O(1)<0$ where $p(t)$ is non-decreasing on $S$, there exists $0<\alpha \leq 1$ such that

$$
\left|\mu^{n}(x+y)-\mu^{n}(x)\right| \leq C \frac{|y|^{\alpha}}{|x|^{1+\alpha}}, \forall|y| \leq \frac{|x|}{2} .
$$

\section{REFERENCES}

[1] A.P. Calderón A. Bellow. A weak type inequality for convolution products. In Chicago Lectures in Math., editor, Harmonic analysis and partial differential equations, pages 41-48, Chicago, IL, 1999. Univ. Chicago Press.

[2] A. Bellow, R.L. Jones, and J.M. Rosenblatt. Almost everywhere convergence of weighted averages. Math.Ann., 294(no. 3):399-426, 1992.

[3] A. Bellow, R.L. Jones, and J.M. Rosenblatt. Almost everywhere convergence of convolution powers. Ergod. Th. ES Dynam, Sys., 14:415-432, 1994.

[4] S.R. Foguel. On iterates of convolutions. Proc. Am. Math. Soc., 47:368-370, 1975.

[5] V. Losert. A remark on almost everywhere convergence of convolution powers. Illinnois J. Math., 43(no. 3):465-479, 1999.

[6] V. Losert. The strong sweeping out property for convolution powers. Ergod. Th. \& Dynam. Sys, 21(no. 1):115-119, 2001.

[7] F. Móricz. Absolutely convergent Fourier series and function classes. J.Math.Anal.Appl., 324(no. 2):1168-1177, 2006. 
[8] V.V. Petrov. Sums of Independent Random Variables. Springer Verlag, New York, Heidelberg, Berlin, 1975.

[9] W.H. Young. On Indeterminate Forms. Proc. London Math. Soc., s2-8(1):40-76, 1910.

[10] A. Zygmund. Trigonometric Series. Cambridge University Press, 1959.

Department of Mathematics And Statistics, University At Albany, 1400 Washington Avenue, Albany, NY, 12222

E-mail address: cw4347@albany.edu 